\title{
A Randomized Controlled Trial Comparing a Mapleson Circuit with Nasal Trumpet to Standard Oxygen Supplementation during EBUS Bronchoscopy under Monitored Anesthesia Care
}

\author{
Wissam Abouzgheib ${ }^{a}$ Talia K. Ben-Jacob ${ }^{b} \quad$ Amit Borah $^{a}$ Rocco Terrigno ${ }^{a}$ \\ Karla Cruz-Morel ${ }^{c}$ Robert Dy ${ }^{b} \quad$ Irwin Gratz ${ }^{b}$ Ziad Boujaoude ${ }^{a}$ \\ anterventional Pulmonary Section, Cooper Medical School at Rowan University, \\ Camden, NJ, USA; ${ }^{b}$ Department of Anesthesiology, Cooper University Hospital, \\ Camden, NJ, USA; ${ }^{C}$ Pulmonary and Critical Care, Palmetto Health, University of \\ South Carolina, Columbia, SC, USA
}

\section{What Is It about?}

Endobronchial ultrasound (EBUS) procedures are often more prolonged than routine bronchoscopies. Some bronchoscopists have responded by performing EBUS in the operating room. At our institution, we perform EBUS in the bronchoscopy suite with monitored anesthesia care for sedation. After noting that the use of a nasal trumpet connected to a Mapleson circuit was an effective intervention for hypoxia, we prospectively compared this method of oxygen supplementation with standard nasal cannula supplementation. A nasal trumpet with a Mapleson circuit was associated with significantly fewer episodes of clinically relevant hypoxia and with shorter procedure times. This simple system for oxygen supplementation could easily be accepted as a standard for EBUS procedures and for other procedures in which there is an increased risk of desaturation.

\section{Keywords}

EBUS procedure $\cdot$ Bronchoscopy $\cdot$ Sedation $\cdot$ Mapleson circuit · Oxygen · Desaturation

\section{Abstract}

Background: Endobronchial ultrasound (EBUS) procedures tend to be longer than routine bronchoscopies. Increased duration and sedative dosing put patients at increased risk for hypoxic events. Objective: To determine whether oxygen supplementation via a nasal trumpet connected to a Mapleson B circuit (NTM) was effective in decreasing hypoxic events when compared with the standard of care, oxygen supplementation with a nasal cannula (NC). Methods: Patients referred for EBUS-guided transbronchial needle aspiration with monitored

This study was registered at ClinicalTrials.gov (identifier: NCT03815097). 
anesthesia care (MAC) were randomized 1:1 to NTM or to NC. Hypoxia-related procedural interruptions, the primary endpoint of the study, were documented for all patients. Patients in the NC group who had refractory desaturations were allowed to cross over to the NTM group. Secondary endpoints included: number of crossovers from NC to NTM, sedative dosing, total procedure times, whether procedure goals were achieved, complications apart from hypoxia, patient discharge status. Results: Fifty-two patients were randomized to NC and 48 to NTM. Baseline characteristics were comparable. The NC group had significantly more interruptions than did the NTM group $(p<0.001)$. Procedure duration was also significantly $(p<$ 0.03 ) shorter for the NTM group. Fourteen patients were crossed over from NC to NTM because of hypoxia. Thirteen out of the 14 completed the procedure with no interruptions. All procedures were successfully completed, and all goals were achieved. All patients returned to baseline status prior to discharge. Three minor complications of epistaxis occurred. Conclusion: For patients undergoing EBUS with MAC, oxygen supplementation with NTM significantly decreased the incidence of hypoxic events when compared with NC. NTM may also be of value for other subsets of patients who are at increased risk for desaturation when undergoing bronchoscopy.

(C) 2019 The Author(s)

Published by S. Karger AG, Basel

\section{Introduction}

Procedure durations for endobronchial ultrasound with transbronchial needle aspiration biopsy (EBUS-TBNA) tend to be longer than those for standard bronchoscopy due (1) to the sampling at times of multiple sites and (2) to the concomitant use of rapid on-site cytology. Duration of sedation and total doses therefore tend to be greater. Both of these factors increase the risk of clinically relevant desaturation during EBUS, and at different institutions responses to this possibility have ranged from EBUS with routine bronchoscopy sedation to EBUS in the operating room (OR) with general anesthesia (GA) [1-3]. At Cooper University Hospital, we have chosen to perform EBUS in the bronchoscopy suite with monitored anesthesia care (MAC) provided by an anesthesiologist. When clinically relevant desaturation does occur, the anesthesiologist needs to control the airway. This often leads to temporary discontinuation and at times to termination of the EBUS procedure. After some initial success with the use of a nasal trumpet connected to a Mapleson circuit (NTM) to correct hypoxia occurring during EBUS with MAC, we instituted a prospective trial comparing NTM throughout the procedure with the current standard for oxygen supplementation, a nasal cannula (NC).

\section{Methods}

\section{Subjects}

The study was approved by the Cooper University Hospital Institutional Review Board (IRB \#14-081). All adult patients (inpatient or outpatient) referred to interventional pulmonary for EBUS for the diagnostic workup of a mass and/or mediastinal adenopathy over the study interval were evaluated for study entry. Exclusion criteria included contraindications for bronchoscopy, uncorrectable coagulopathy, known nasal passage obstruction or severe epistaxis, history of intolerance of MAC, pregnancy, and inability to obtain informed consent. 


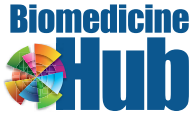

Fig. 1. Mapleson circuit during a procedure.

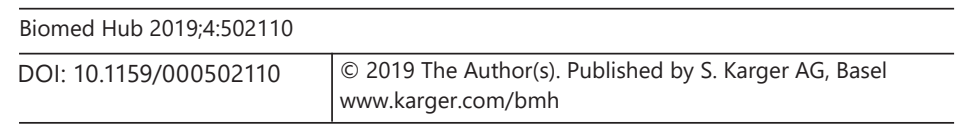

Abouzgheib et al.: A Randomized Controlled Trial Comparing a Mapleson Circuit with Nasal Trumpet to Oxygen Supplementation during EBUS Bronchoscopy under MAC

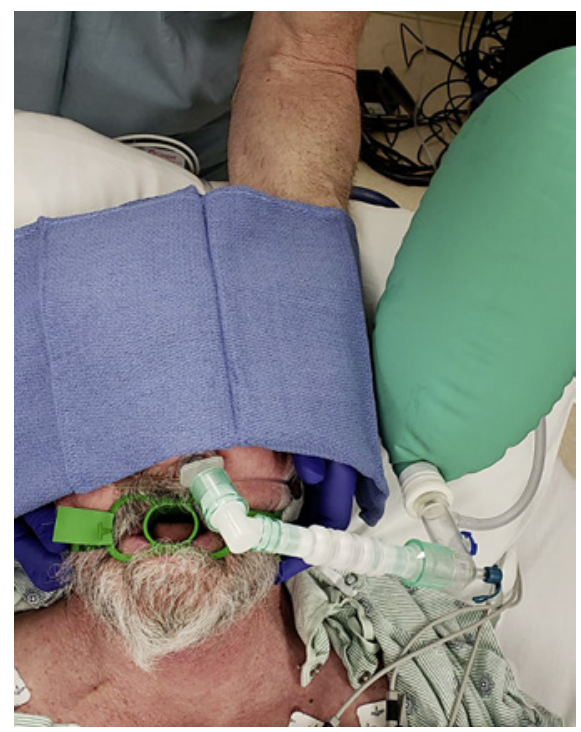

\section{Study Design}

This was a prospective randomized controlled study of two different approaches to oxygen supplementation during MAC for EBUS. Informed consent was obtained in all cases. Subjects have given their written informed consent to publication of images. Randomization was achieved using computer software. The control group received supplemental oxygen via an NC titrated as needed to a maximum of $10 \mathrm{~L}$, our standard approach. The study group received oxygen via a Mapleson circuit connected to a nasal trumpet (NTM). Figure 1 demonstrates the NTM system in place for a procedure. Apart from modality of oxygen supplementation, all procedural details were identical for both groups.

An anesthesiologist administered MAC, monitored vital signs, and performed any airway interventions. All procedures were performed by one of two interventional pulmonologists (W.A. and Z.B.). EBUS-TBNA was performed using an Olympus bronchoscope (BF-UC-180F) and 21-gauge needles. In order to minimize procedural delays, our standard protocol involves the use of two needles for each patient. At least 3 aspirations were obtained from each target. Procedure time was defined as time from initial introduction of the bronchoscope to final withdrawal. Rapid on-site examination was available for all procedures.

The primary endpoint of the study was the number of hypoxia-related interruptions that occurred during the procedure. For this study, hypoxia was defined as a drop in saturation of $5 \%$ or more that did not self-correct. An interruption was defined as a cessation of bronchoscopist activities related to the need to intervene to improve oxygenation. Interventions included the addition of another oxygen source such as a $100 \%$ non-rebreather, a jaw-thrust maneuver to open up the upper airway, and the need for bag mask ventilation. Some interruptions included the need to completely withdraw the bronchoscope from the airway while others did not.

The protocol included a crossover pathway. Crossover from the NC group to the NTM protocol was allowed for patients under either of two conditions: (1) a patient experienced 4 or more interruptions within 5 consecutive minutes, or (2) recovery had not occurred within 2 min of an interruption. For patients who did not tolerate either arm of the study, the plan was to terminate the procedure and reschedule it in the OR under GA.

Secondary endpoints included amounts of sedatives administered, number of crossovers from the NC arm to the NTM arm, procedure time, whether or not procedure goals were achieved, status of the patient at time of discharge, and incidence of nosebleed. 
Table 1. Baseline characteristics

\begin{tabular}{llll}
\hline & $\begin{array}{l}\text { NC group } \\
(n=52)\end{array}$ & $\begin{array}{l}\text { NTM group } \\
(n=48)\end{array}$ & $p$ value \\
\hline Age (SD), years & $61.52(11.46)$ & $63.92(10.68)$ & 0.28 \\
Males:females & $28: 24$ & $27: 21$ & 0.8 \\
Race & & & 0.2 \\
$\quad$ White & 37 & 41 & \\
$\quad$ Black & 9 & 5 & 0.33 \\
$\quad$ Other & $69.2(6.41)$ & $27.92(6.81)$ & 0.44 \\
BMI (SD) & 2 & 2 & 0.93 \\
Mallampati score & 30 & 31 & 0.64 \\
Smoking, pack-years & 7 & 5 & 0.07 \\
Hx of OSA & 98 & 99 & 0.53 \\
Baseline SaO & & & \\
Sedation & 1 & 1.5 & \\
$\quad$ Versed, $\mu$ r & 50 & 53 & \\
$\quad$ Fentanyl, mg & 400 & 40 & \\
$\quad$ Propofol, mg & 40 & 39 & \\
$\quad$ Ketamine, mg & & & \\
\hline
\end{tabular}

Values for Mallampati score, smoking, baseline $\mathrm{SaO}_{2}$, and sedation are expressed as medians.

Table 2. Biopsy sites and diagnoses

\begin{tabular}{lr}
\hline Sites biopsied & \\
Lymph nodes & 203 \\
Masses & 12 \\
Left adrenal gland & 1 \\
\hline Final diagnoses & \\
NSCLC & 46 \\
Nonspecific adenopathy (staging) & 28 \\
Sarcoidosis & 15 \\
B-cell lymphoma & 3 \\
Small cell carcinoma & 3 \\
Carcinoid & 2 \\
Melanoma & 2 \\
Metastatic cervical cancer & 1 \\
Total & 100 \\
\hline
\end{tabular}

\section{Statistical Analysis}

The Mann-Whitney U test was used to determine statistical significance for the interruptions between groups. $t$ testing and Pearson's $\chi^{2}$ test were used to analyze demographics of both groups. A $p$ value of $<0.05$ was considered to be statistically significant.

\section{Results}

Between March 2016 and April 2018, 148 patients were screened for eligibility. One hundred patients met eligibility criteria and were randomized, 52 to NC and 48 to NTM. Both groups were well balanced with respect to baseline characteristics, including gender, Mallampati scores, and smoking status (Table 1). The incidence of obstructive sleep apnea did not differ between groups. There was no significant difference in baseline room air oxygen saturation levels. 
Abouzgheib et al.: A Randomized Controlled Trial Comparing a Mapleson Circuit with Nasal Trumpet to Oxygen Supplementation during EBUS Bronchoscopy under MAC

MAC was achieved with combinations of versed, fentanyl, propofol, and ketamine. Both groups received comparable amounts of all four sedatives (see Table 1). Table 2 lists sites biopsied and final diagnoses. Two hundred and three lymph nodes were biopsied, 12 lung masses were accessed with the convex EBUS bronchoscope, and one left adrenal gland was biopsied with the EBUS bronchoscope introduced through the esophagus. (All patients underwent EBUS procedures, and 1 patient underwent both EBUS and esophageal ultrasound [EUS].) Primary lung cancer was the most common diagnosis.

In the NC group, 41 out of 52 patients (79\%) had interruptions, with a mean of 2.6 and a range of 1-6. In the NTM group, 1 patient (2\%) had 2 interruptions, while the remaining 47 patients underwent EBUS-TBNA without any interruptions. The difference between the two groups was significant, with $p<0.001$. Procedure duration was also shorter for the NTM group (27.25 $\pm 10.08 \mathrm{~min}$ ) versus the NC group (31.98 $\pm 11.29 \mathrm{~min})$, with $p<0.03$.

Fourteen patients from the NC group were crossed over to NTM. Three out of the 14 were crossed over after the first interruption, as they had not recovered within 2 min of standard airway maneuvers. Three patients were crossed over after a second interruption, 6 patients after 3 interruptions, and 4 were crossed over after 4 interruptions. Once crossed over, 13 out of the 14 patients completed the procedure without any subsequent interruptions. The remaining patient had one additional interruption after crossing over to NTM, but the interruption did not prevent procedure completion. All baseline characteristics apart from gender were similar when comparing the crossover group to the group that did not cross over; 10 out of $14(71 \%)$ of crossover patients were women, whereas 14 out of $38(37 \%)$ in the noncrossover group were women $(p=0.026)$.

All patients enrolled in this study were able to complete their planned procedures; no cancellations occurred. There were no major complications directly related to the study. All patients in both arms returned to preprocedure status prior to discharge. There were no unplanned admissions, and no inpatient required escalation of care. Three minor complications occurred in this study, all nosebleeds related to insertion of the nasal trumpet. Two patients in the initial NTM group and 1 patient in the crossover group suffered from epistaxis when the nasal trumpet was inserted. One episode of epistaxis resolved spontaneously. The other two resolved after local instillation of lidocaine/epinephrine.

\section{Discussion}

Bronchoscopy and transbronchial needle aspiration biopsy using the convex curvilinear ultrasound bronchoscope (EBUS-TBNA) and, to a lesser extent, EUS and fine needle aspiration with the same bronchoscope have become established and important diagnostic tools in the hands of the interventional pulmonologist faced with mediastinal adenopathy and with masses adjacent to the airways and/or esophagus [4,5]. EBUS procedures tend to be longer than standard bronchoscopies due to the acquisition of multiple targets when staging lung cancer and to the frequent use of on-site cytology. In addition, patients undergoing EBUS bronchoscopy for suspected lung cancer often have coexisting comorbidities such COPD [6], making them more prone to intraprocedural hypoxic episodes. Because of these issues, there has been an ongoing discussion about optimal procedure location and sedation modalities for EBUS, ranging from EBUS in the bronchoscopy suite with standard moderate sedation to EBUS in the bronchoscopy suite with MAC administered by an anesthesiologist to EBUS in the OR with GA [1-3]. The first two require spontaneous ventilation, increase the risk of hypoxemia, and may need to have the procedure terminated for airway control when adverse events do occur. EBUS in the OR with GA allows better control of oxygenation but involves the use of a laryngeal mask airway or an endotracheal tube. Both 


\section{Biomenticicine 絇而b}

Fig. 2. Nasal trumpet and velopharynx.

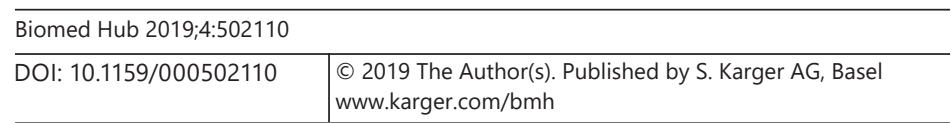

Abouzgheib et al.: A Randomized Controlled Trial Comparing a Mapleson Circuit with Nasal Trumpet to Oxygen Supplementation during EBUS Bronchoscopy under MAC

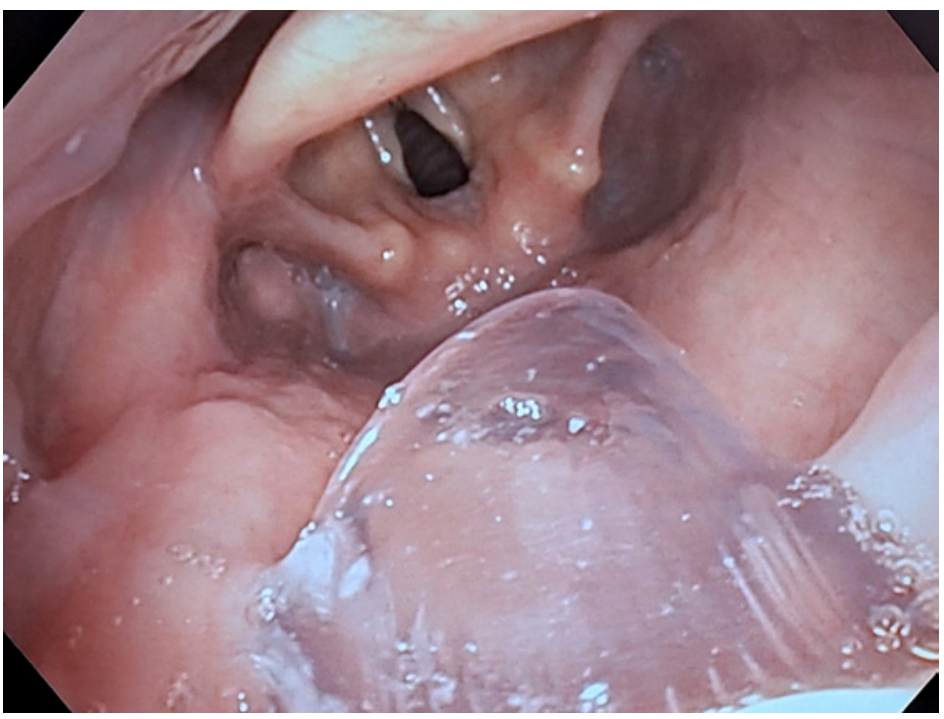

significantly limit the lumen available for bronchoscopy, a potential problem, and EUS cannot be performed with a laryngeal mask airway in place. In addition, GA in the OR incurs significantly higher costs with no ostensible increase in yield or patient satisfaction $[2,3$, 7]. MAC has been used for years for gastrointestinal endoscopy, with a relatively large body of related literature. At our institution, MAC is now the standard of care for anesthesia when performing EBUS.

Hypoxia is probably the most common complication of bronchoscopy; a definitive statement is difficult, as the literature varies markedly with respect to the definition and documentation of hypoxia. A recent extensive review lists hypoxia as a complication in $0.7-76.3 \%$ of cases, a huge range that more likely reflects definition and documentation than true differences in incidence [8]. Two studies that looked specifically at rates of desaturation with EBUS under MAC yielded rates of 36 and 45\% [1, 9].

The best argument for EBUS in the OR is that it affords better airway control (despite the issues involved) and is more effective in preventing respiratory decompensations and the need for procedure interruption. In this prospective study, we have demonstrated that the NTM system can allow effective completion of EBUS under MAC in the bronchoscopy suite with a very low incidence of hypoxic events; events occurred in $2 \%$ of patients in the NTM group. If one adds to the NTM group those patients crossed over to NTM, events occurred in $4 \%$ of NTM patients. Both of these are far lower than the rates of 36 and $45 \%$ cited above [1, 9]. The decreased incidence of hypoxic events and thus of interruptions translated directly into increased efficiency in the form of decreased procedure durations. One can conclude based upon these study data that with the use of NTM most patients can be safely and efficiently diagnosed in the bronchoscopy suite.

Sedation-induced hypoxia is related to two main phenomena: (1) central depression of respiratory drive, and (2) upper airway obstruction caused by relaxation of the velopharyngeal muscles and the tongue $[10,11]$. (During bronchoscopy, these factors are potentially exacerbated by airway obstruction caused by the bronchoscope, suctioning of inspired gasses, and instillation of fluids into the airways.) Insertion of a nasal trumpet allows bypass of the upper airway obstruction (see Fig. 2), and the use of a Mapleson circuit allows the anesthesiologist to increase both flow and inspired $\mathrm{FIO}_{2}$. The efficacy of NTM as an intervention documented in this study leads one to conclude that upper airway collapse is the dominant mechanism of hypoxemia with levels of sedation up to and including MAC. 


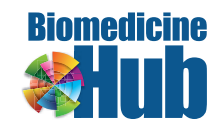

\begin{tabular}{|c|c|}
\hline \multicolumn{2}{|l|}{ Biomed Hub 2019:4:502110 } \\
\hline DOI: $10.1159 / 000502110$ & $\begin{array}{l}\text { (c) } 2019 \text { The Author(s). Published by S. Karger AG, Basel } \\
\text { www.karger.com/bmh }\end{array}$ \\
\hline
\end{tabular}

Abouzgheib et al.: A Randomized Controlled Trial Comparing a Mapleson Circuit with Nasal Trumpet to Oxygen Supplementation during EBUS Bronchoscopy under MAC
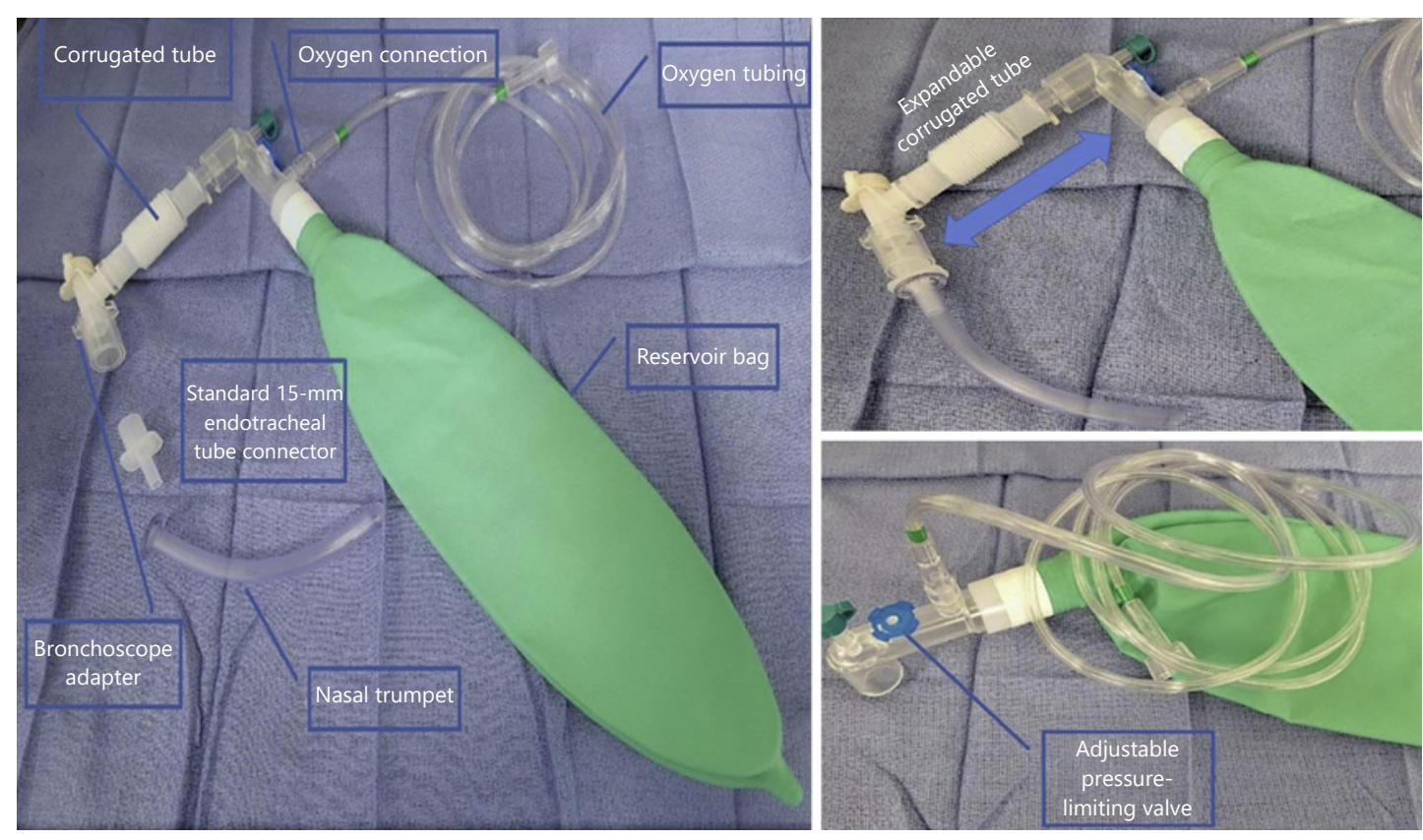

Fig. 3. Components of a Mapleson circuit.

Fig. 4. Nasal trumpet above vocal cords.

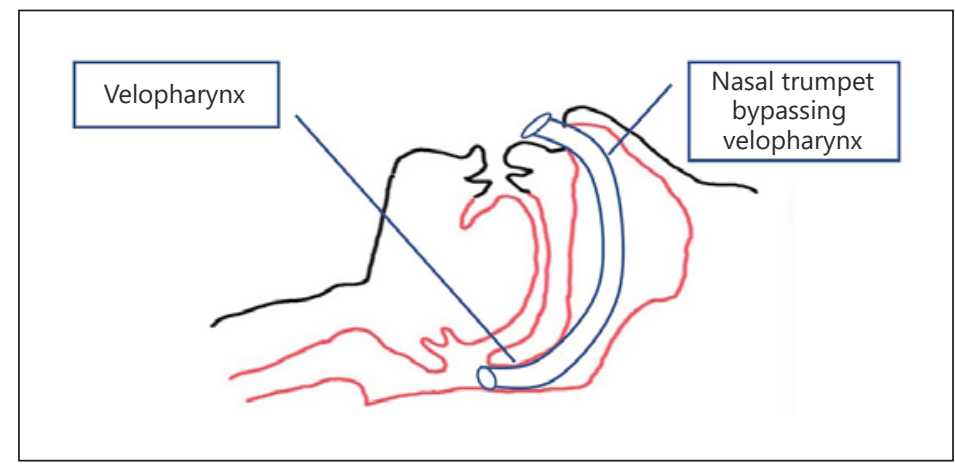

A Mapleson circuit is a breathing circuit that is an assembly of components; it includes a reservoir bag, corrugated tubing, and an adjustable pressure-limiting valve (see Fig. 3). This circuit connects the patient's airway to fresh gas flow (oxygen in this study) and allows spontaneous, assisted, or controlled respiration. It is lightweight, inexpensive, and simple to use. At our hospital the cost of a Mapleson B circuit is USD 4.86. During spontaneous inspiration, oxygen flows from the oxygen source and reservoir to the patient. During expiration, initial expiratory flow fills the corrugated tubing and interrupts the forward flow of gasses. This allows the reservoir to refill. When the reservoir bag is full, the adjustable expiratory valve opens and vents remaining expired gas into the atmosphere. With inspiration, the vent closes. Inspired gas includes the gas in the tubing, gas from the reservoir, and gas flowing directly from the oxygen source. Whereas initial exhaled gas consisted of dead space ventilation which had not participated in gas exchange, all inspired gasses contain $100 \%$ oxygen [12]. Performance of the circuit and classification are determined by the location of the components in the Mapleson circuit. We used the Mapleson B circuit in this study as that is the circuit available in our institution. The Mapleson B system features the fresh gas inlet near the patient, distal to the expiratory valve. Typically, Mapleson circuits are attached either to a face mask or to 
an endotracheal tube. Different from conventional practice, in our study the Mapleson circuit was connected to a nasal trumpet. The distal end of the nasal trumpet sat above the vocal cords, delivering high-flow oxygen and assisting in removal of exhaled $\mathrm{CO}_{2}$ while the patient was breathing spontaneously (Fig. 4).

Goudra et al. [11] have previously described the use of NTM during gastrointestinal endoscopy, but their account is anecdotal; we are unaware of any published series in that literature. To our knowledge, this report represents the first published series and the first report of the use of an NTM system for bronchoscopy.

This study was notable for a very high incidence of hypoxia in the NC group, with interruptions occurring in 79\% (41 out of 52). This rate is higher than many previously reported rates $[1,8,9]$. The rate may be due to the fact that a combination of 4 different sedatives was used in our study. However, there was no difference in sedatives administered when comparing both groups. End-tidal carbon dioxide was not used as a parameter in this study because even though it was monitored, we were unsure of the accuracy of the numbers given the high fresh gas flow used with the Mapleson circuit.

The main limitation of the study is the fact that it was not blinded to the operators, as this was not possible. In order to try to eliminate operator bias, we clearly defined desaturation episodes, allowed time for self-correction of transient hypoxias, and, more importantly, clearly defined the airway interventions and maneuvers that led to interruption of a bronchoscopy.

In summary, the use of a Mapleson circuit with nasal trumpet is a simple, safe, and effective way to provide supplemental oxygen during EBUS and to significantly decrease the incidence of hypoxia and the associated procedural interruptions. While this study was confined to EBUS with MAC, there is no reason why NTM cannot be applied to bronchoscopy with conscious sedation or MAC performed on any patient deemed at high risk for hypoxia; known sleep apnea, morbid obesity, severe COPD, and anticipation of a prolonged procedure might be reasonable criteria for the preemptive use of NTM.

\section{Statement of Ethics}

Subjects (or their parents or guardians) have given their written informed consent. The study protocol has been approved by the research institute's committee on human research.

\section{Disclosure Statement}

The authors declare no conflicts of interest.

\section{Funding Sources}

No funding was obtained for this study.

\section{Author Contributions}

All authors contributed equally to protocol creation, execution, patient enrollment, data collection and analysis and writing of the manuscript. 
Abouzgheib et al.: A Randomized Controlled Trial Comparing a Mapleson Circuit with Nasal Trumpet to Oxygen Supplementation during EBUS Bronchoscopy under MAC

\section{References}

1 Douglas N, Ng I, Nazeem F, Lee K, Mezzavia P, Krieser R, et al. A randomised controlled trial comparing highflow nasal oxygen with standard management for conscious sedation during bronchoscopy. Anaesthesia. 2018 Feb;73(2):169-76.

2 Casal RF, Lazarus DR, Kuhl K, Nogueras-González G, Perusich S, Green LK, et al. Randomized trial of endobronchial ultrasound-guided transbronchial needle aspiration under general anesthesia versus moderate sedation. Am J Respir Crit Care Med. 2015 Apr;191(7):796-803.

3 Canneto B, Ferraroli G, Falezza G, Infante MV. Ideal conditions to perform EBUS-TBNA. J Thorac Dis. 2017 May; 9 Suppl 5:S414-7

4 Dooms C, Tournoy KG, Schuurbiers O, Decaluwe H, De Ryck F, Verhagen A, et al. Endosonography for mediastinal nodal staging of clinical N1 non-small cell lung cancer: a prospective multicenter study. Chest. 2015 Jan; 147(1):209-15.

5 Vilmann P, Clementsen PF. Combined EUS and EBUS are complementary methods in lung cancer staging: do not forget the esophagus. Endosc Int Open. 2015 Aug;3(4):E300-1.

6 Young RP, Hopkins RJ, Christmas T, Black PN, Metcalf P, Gamble GD. COPD prevalence is increased in lung cancer, independent of age, sex and smoking history. Eur Respir J. 2009 Aug;34(2):380-6.

7 Khan A, Carillo C, Zamel R, Czarnecka K, Darling G, Pierre A, et al. EBUS-TBNA and choice of sedation: a comparison between general and conscious sedation performed in OR and OPD settings respectively and cost implications. Chest. 2014;146:749A.

8 Leiten EO, Martinsen EM, Bakke PS, Eagan TM, Grønseth R. Complications and discomfort of bronchoscopy: a systematic review. Eur Clin Respir J. 2016 Nov;3:33324.

9 Vila E, Mases A, Fernandez JL, Vela E, Gallart L. Anesthesia technique and patient and bronchoscopist satisfaction during endobronchial ultrasound-guided transbronchial needle aspiration performed under conscious sedation. Eur J Anaesthesiol. 2011;28:78.

10 Hillman DR, Platt PR, Eastwood PR. Anesthesia, sleep, and upper airway collapsibility. Anesthesiol Clin. 2010 Sep;28(3):443-55.

11 Goudra B, Singh PM. Airway Management During Upper GI Endoscopic Procedures: State of the Art Review. Dig Dis Sci. 2017 Jan;62(1):45-53.

12 Kaul TK, Mittal G. Mapleson's breathing systems. Indian J Anaesth. 2013 Sep;57(5):507-15. 